\title{
Live Cell-Based Sensor Devices
}

\author{
Junko Okuda-Shimazaki, Ken-Ichi Wada and Akiyoshi Taniguchi*
}

\author{
Cell-Sensing Group, Biomaterials Center, National Institute for Materials Science, 1-1 Namiki, Tsukuba, Ibaraki 305- \\ 0044, Japan
}

\begin{abstract}
Living cells maintain life functions by responding quickly and with great sensitivity to changes in the external environment. Consequently, sensors using cells as active elements are thought to be able to perform analyses faster and with more sensitivity than previously possible. Cell-based sensors can be roughly divided into two types. The first uses microorganisms such as Escherichia coli or yeast as active elements (Microbial cells). The second type uses human and animal cells (Mammalian cells). The first type can be cultivated rather easily and has the advantages of being inexpensive and portable. The second type is more complex but has the advantage of potentially being used with human subjects. Most research in this area is concentrated on the first type, microbial sensors, but research on sensors that use mammalian cells has recently become more widespread.

We are exploring the modification of mammalian cells using genetic engineering techniques and developing nextgeneration cell sensors that can visually represent specific reactions. Recently, few researchers, including our group, have successfully produced devices from sensor cells that can process a variety of specimens using MEMS, NEMS, and other nano/micro processing technologies. In this review, we will introduce our research and describe recent trends in this field.
\end{abstract}

\section{MAMMALIAN CELL SENSORS}

The majority of cell-based sensor research involves the use of microorganisms. In recent years, research has started investigating the use of human and animal cells for sensors. We will divide our look at global trends into research using mammalian cells.

When compared with microbial research, research on sensors using animal cells is scarce, but has been slowly increasing since 2000 [1]. Research in this area can be generally broken down into methods that cultivate living animal cells on sensors and methods that use molecular, protein or gene probes. Researchers are attempting to use the former method to detect cellular metabolites directly with sensors and thus detect cell growth [2]. Analyses of various substances are being tested using the latter method [3,4], which has been used to measure inorganic ions, glucose and other substances inside cells.

Additional research projects are working on cell sensors that use fluorescence resonance energy transfer (FRET) within specific proteins (signal transduction-related proteins, for example). This method is promising because it can make observations in real time at the single-cell level. Measurements of calcium and cAMP within cells are being attempted with this method [5-6]. Further research examples include experiments with immunoassays and other tests using live immune cells [7] and trials cultivating cells in microarrays for use as sensors [8].

Methods are being developed that cultivate living animal cells on sensor surfaces and directly analyze metabolic substances generated by drugs or other stimuli. Groups led by Professor Haruyama at the Kyushu Institute of Technology

*Address correspondence to this author at the Cell Engineering Technology Group, Biomaterials Center, National Institute for Materials Science, 1-1 Namiki, Tsukuba, Ibaraki 305-0044, Japan; Tel: +81-29-860-4505; Fax: +81-29-860-4714; E-mail: TANIGUCHI.Akiyoshi@nims.go.jp and Professor Aizawa at the Tokyo Institute of Technology are developing sensors that detect nitric monoxide and nitric oxide using macrophage-like cell strains and endothelial cells [9-12]. Because the metabolites produced by cells under stimulation are miniscule, an important hurdle for the researchers is how to increase sensor sensitivity. An Osaka Prefecture University group led by Professor Sugimoto has been attempting to use cells that visualize various intracellular microstructure proteins as cell sensors [13]. With this method, the group has been able to make detailed analyses by using live cells to observe the effector sites of known drugs and to analyze the actions of unknown drugs based on changes in microstructure, although such methods require the establishment of a quantitative index. Professor Yoshizato's group has cultivated mast cells on an SPR sensor chip as part of the development of a method for real-time detection of physiological changes [14].

\section{LIVE CELL-BASED SENSOR USING GENE TECH- NOLOGY}

In our laboratory, researchers are using molecular biology techniques to fabricate next-generation sensor cells that will detect changes in gene expression in response to drugs and other external stimuli. Using these cells, the aim is to devise a biosensor that can monitor cell stimuli simply and chronologically. Our group has established a stress-response domain in the heat shock protein 70B' (HSP70B') gene and, using this functional domain with reporter genes, has detected cadmium chloride and other cellular toxins. As HSP70 genes are up-regulated in response to a wide-range of cytotoxic stimuli [15-17], cells transfected with reporter vector derived from the HSP70B' promoter can be used as intelligent cytotoxicity sensors (i.e., sensor cells) [18-20]. We demonstrated that this method has about four times the sensitivity of existing methods [18]. We have already found the cytotoxic responding element (CRE) in HSP70B' gene promoter, in which functional HSEs are contained and AP-1 site is removed by base substitution from AGT to CAG. The 
transfection of luciferase constructs driven by modified HSP70B' promoter, which contains tandem repeats of CRE in front of the HSP70B' promoter resulted in fabrication of highly sensitive cytotoxicity sensor cells [19]. Furthermore, we have succeeded improvement in sensitivity of live cellbased sensor cells by co-transfection of a reporter gene driven by a modified HSP70B' and HSF1 expression vector [20]. Using GFP as a receptor gene, it is possible to chronologically detect cellular toxins such as cadmium chloride (Fig. 1). Because the HSP70 gene responds to numerous types of stressor, we expect that this functional domain can be used for very sensitive detection of a wide range of cellular toxins.
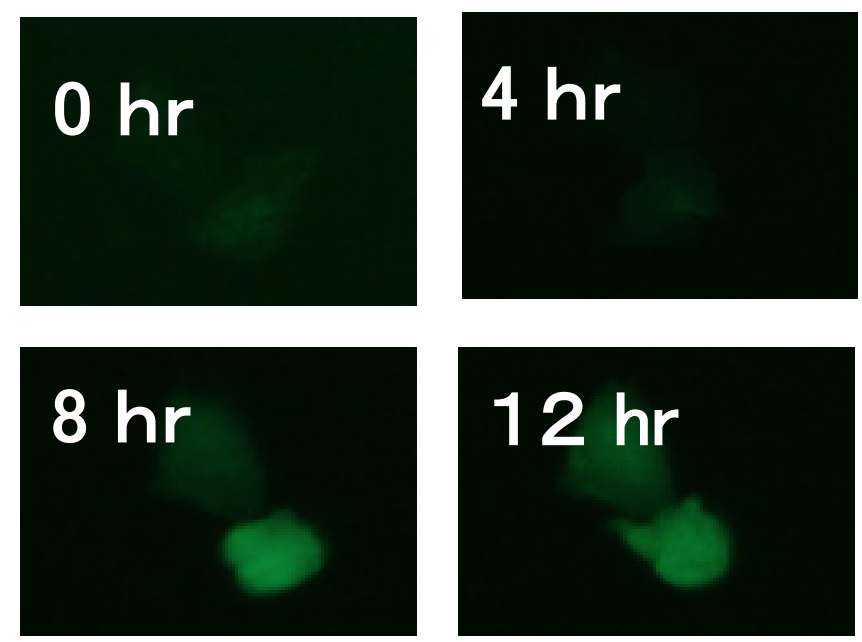

Fig. (1). Chronological detection of cell toxicity with sensor cells.

\section{CONVERGENCE OF SENSOR CELLS AND MEMS TECHNOLOGY}

Microfluidic devices perform rapid and high-throughput measurements on small sample volumes. A Tohoku University group led by Professor Matsunaga has embarked on a unique research project that incorporates nerve cells in microchannel analysis [21]. Many other researchers have developed microfluidic cell culture methods for analyzing cell functions or metabolism [1,22-24]. To analyze cellular responses, Thompson et al. have developed a microfluidic platform for continuous monitoring of gene expression in live cells by profiling the activation of the transcription factor NF-kappaB in HeLa S3 cells in response to varying doses of the inflammatory cytokine TNF-alpha [25]. And they have demonstrated that the kinetics and magnitude of induced fluorescence in the reporter cell lines can be further improved to maximize the fluorescence readout from reporter cell lines using reporter cell line subcloning and cell cycle synchronization, thereby improving their applicability to live cell expression profiling [26].

We have experimented with cultivating our sensor cells in microwells and microchannels with the idea of applying them in cell arrays and other high-throughput analytical methods. These experiments showed that it is possible to cultivate sensor cells in microwells and microchannels (Fig. 2). The master mold for the microfluidic channels was fabricated using a rapid proto-typing method utilizing a simple photopolymerization procedure adapted from a modified liquid-crystal-display projector, which can easily generate patterns on a PC monitor and project the images with $5 \mu \mathrm{m}$ - resolution [27-30]. This mask-less irradiation procedure allows the creation of any 2-dimentional design in the master mold without the need for specific skills and equipment, thereby saving time and money, and providing a powerful tool to achieve custom designed microfluidic systems. We expect that it will be possible to use sensor cells like these to develop next-generation devices capable of detecting cellular toxicities with a high throughput in microwells and microchannels.

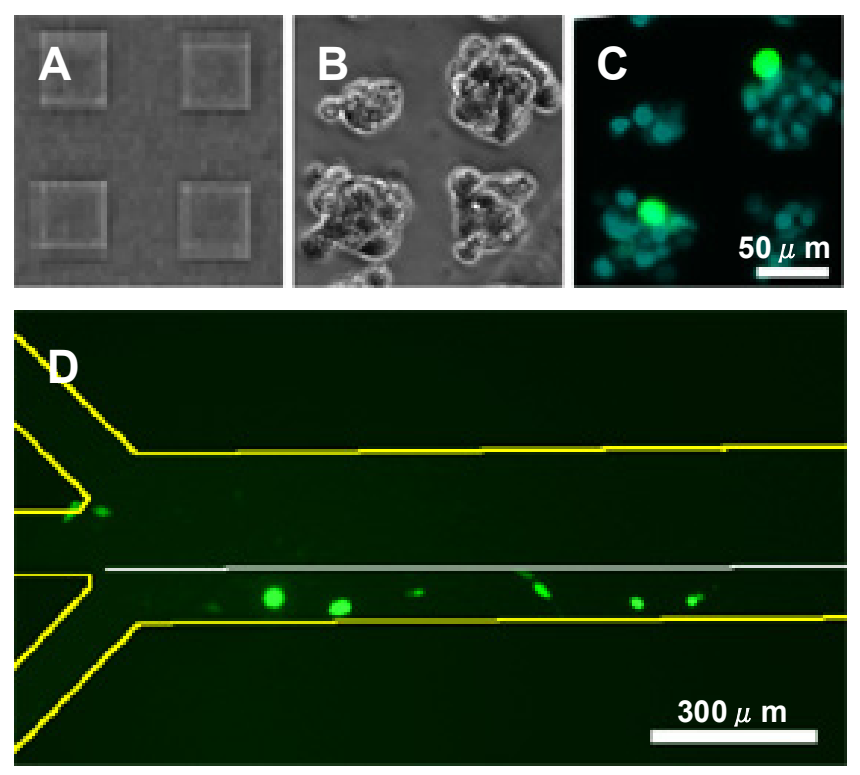

Fig. (2). Convergence of sensor cells and MEMS technology. A: cell adhesion domains. B: micropatterned sensor cells. C: GFP expression of micropatterned sensor cells. D: sensor cells in a microchannel.

\section{FUTURE OUTLOOK}

We expect the main body of research to focus on developing next-generation measurements capable of processing multiple specimens through convergence with technologies from other fields (such as molecular biology and nanotechnology).

To improve the sensitivity, specificity, and specimenprocessing capability of sensors, however, we feel that it is necessary to adopt technology from other fields, particularly nanotechnology and biotechnology.

\section{CONCLUSION}

Live cell-based sensors may potentially be used as an examination technology in medical field, as well as for celltoxicity inspection of medical supplies, nanomaterials, biomaterials, environmental factors and other materials. With the convergence of biotechnology and nanotechnology, even better measurement technologies are expected to be established and extended into vital nano-bio fields

\section{REFERENCES}

[1] El-Ali J, Sorger PK, Jensen KF. Cells on chips. Nature 2006; 442: 403-11.

[2] Pescheck M, Schrader J, Sell D. Novel electrochemical sensor system for monitoring metabolic activity during the growth and cultivation of prokaryotic and eukaryotic cells. Bioelectrochemistry 2005; 67: 47-55. 
[3] Schar-Zammaretti P, Ziegler U, Forster I, Groscurth P, SpichigerKeller UE. Potassium-selective atomic force microscopy on ionreleasing substrates and living cells. Anal Chem 2002; 74: 4269-74.

[4] Xu H, Aylott JW, Kopelman R. Fluorescent nano-PEBBLE sensors designed for intracellular glucose imaging. Analyst 2002; 127: 1471-7.

[5] Azpiazu I, Gautam N. A fluorescence resonance energy transferbased sensor indicates that receptor access to a $\mathrm{G}$ protein is unrestricted in a living mammalian cell. J Biol Chem 2004; 279: 2770918.

[6] Rich TC, Karpen JW. Review article: Cyclic AMP sensors in living cells: What signals can they actually measure? Ann Biomed Eng 2002; 30: 1088-99.

[7] Pizziconi VB, Page DL. A cell-based immunobiosensor with engineered molecular recognition 1 . Design feasibility. Biosens Bioelectron 1997; 12: 287-99.

[8] Taylor LC, Walt DR. Application of high-density optical microwell arrays in a live-cell biosensing system. Anal Biochem 2000; 278: $132-42$.

[9] Kamei K, Haruyama T, Mie M, Yanagida Y, Aizawa M, Kobatake E. The construction of endothelial cellular biosensing system for the control of blood pressure drugs. Biosens Bioelectron 2004; 19: $1121-4$.

[10] Kamei K, Haruyama T, Mie M, Yanagida Y, Aizawa M, Kobatake E. Development of immune cellular biosensing system for assessing chemicals on inducible nitric oxide synthase signaling activator. Anal Biochem 2003; 320: 75-81.

[11] Kamei K, Haruyama T, Mie M, Yanagida Y, Kobatake E, Aizawa $\mathrm{M}$. Cellular biosensing system for assessing immunomodulating effects on the inducible nitric oxide synthase (iNOS) cascade. Biotechnol Lett 2003; 25: 321-5.

[12] Haruyama T. Micro- and nanobiotechnology for biosensing cellular responses. Adv Drug Deliv Rev 2003; 55: 393-401.

[13] Sugimoto K, Urano T, Zushi $\mathrm{H}$, et al. Molecular dynamics of aurora-A kinase in living mitotic cells simultaneously visualized with histone $\mathrm{H} 3$ and nuclear membrane protein importin alpha. Cell Struct Funct 2002; 27: 457-67.

[14] Hide M, Tsutsui T, Sato H, et al. Real-time analysis of ligandinduced cell surface and intracellular reactions of living mast cells using a surface plasmon resonance-based biosensor. Anal Biochem 2002; 302: 28-37.

[15] Kiang JG, Tsokos GC. Heat shock protein $70 \mathrm{kDa}$ : Molecular biology, biochemistry, and physiology. Pharmacol Ther 1998; 80: 183201.

[16] Schlesinger MJ. Heat-Shock Proteins. J Biol Chem 1990; 265: 12111-4.
[17] Okuda-Shimazaki J, Yamamoto A, Kuroda, D, Hanawa T, Taniguchi A. The effect of metal materials on heat shock protein 70B' gene expression. Open Biotechnol J 2007; 1: 14-17.

[18] Wada KI, Taniguchi A, Xu LM, Okano T. Rapid and highly sensitive detection of cadmium chloride induced cytotoxicity using the HSP70B ' promoter in live cells. Biotechnol Bioeng 2005; 92: 4105.

[19] Wada KI, Taniguchi A, Okano T. Highly sensitive detection of cytotoxicity using a modified HSP70B' promoter. Biotechnol Bioeng 2007; 97: 871-876.

[20] Wada KI, Okuda J, Taniguchi A. Improvement of sensitivity of live cell-based sensor cells by co-transfection of reporter gene driven from modified HSP70B' and HSF1 expression vector. Open Biotechnol J 2007; 1: 21-25.

[21] Kaji H, Nishizawa M, Matsue T. Localized chemical stimulation to micropatterned cells using multiple laminar fluid flows. Lab Chip 2003; 3: 208-11.

[22] Weber N, Caliebe J, Ziemer G, Wendel HP. Material-dependent levels of heat-shock protein 70 (hsp70) in human plasma following contact of blood with artificial surfaces. J Biomater Sci Polym Ed 2003; 14: 747-60.

[23] Beebe D, Wheeler M, Zeringue H, Walters E, Raty S. Microfluidic technology for assisted reproduction. Theriogenology 2002; 57 : 125-35.

[24] Park TH, Shuler ML. Integration of cell culture and microfabrication technology. Biotechnol Progr 2003; 19: 243-53.

[25] Thompson DM, King KR, Wieder KJ, Toner M, Yarmush ML, Jayaraman A. Dynamic gene expression profiling using a microfabricated living cell array. Anal Chem 2004; 76: 4098-103.

[26] Wieder KJ, King KR, Thompson DM, Zia C, Yarmush ML, Jayaraman A. Optimization of reporter cells for expression profiling in a microfluidic device. Biomed Microdevices 2005; 7: 213-22

[27] Kobayashi J, Yamato M, Itoga K, Kikuchi A, Okano T. Preparation of microfludic devices using micropatterning of a phtosensitive material by a maskless, liquid-crystal-display projection method. Adv Mater 2004; 16: 1997-2001.

[28] Itoga K, Kobayashi J, Yamato M, Kikuchi A, Okano T. Maskless liquid-crystal-display projection photolithography for improved design flexibility of cellular micropatterns. Biomaterials 2006; 27: 3005-3009.

[29] Itoga K, Yamato M, Kobayashi J, Kikuchi A, Okano T. Micropatterned surfaces prepared using a liquid crystal projector-modified photopolymerization device and microfluidics. J Biomed Mater Res A 2004; 69: 391-397.

[30] Itoga K, Yamato M, Kobayashi J, Kikuchi A, Okano T. Cell micropatterning using photopolymerization with a liquid crystal device commercial projector. Biomaterials 2004; 25: 2047-2053. 